Cahiers d'études italiennes

$5 \mid 2006$

NOVECENTO... E DINTORNI

Images littéraires de la société contemporaine (2)

\title{
La femme et la mer dans Il silenzio de Francesco Biamonti : au-delà de la séduction
}

Matteo Meschiari

\section{OpenEdition}

\section{Journals}

Édition électronique

URL : http://journals.openedition.org/cei/805

DOI : $10.4000 /$ cei.805

ISSN : 2260-779X

Éditeur

UGA Éditions/Université Grenoble Alpes

Édition imprimée

Date de publication : 15 septembre 2006

Pagination : 11-21

ISBN : 978-2-84310-086-4

ISSN : 1770-9571

\section{Référence électronique}

Matteo Meschiari, « La femme et la mer dans // silenzio de Francesco Biamonti : au-delà de la séduction », Cahiers d'études italiennes [En ligne], 5 | 2006, mis en ligne le 15 mars 2008, consulté le 28 mars 2021. URL : http://journals.openedition.org/cei/805 ; DOI : https://doi.org/10.4000/cei.805 


\title{
LA FEMME ET LA MER \\ DANS IL SILENZIO DE FRANCESCO BIAMONTI
}

\author{
AU-DELÀ DE LA SÉDUCTION
}

\section{Matteo Meschiari}

Université Charles de Gaulle-Lille 3

La nature est un temple où de vivants piliers Laissent parfois sortir de confuses paroles.

BAUDELAIRE

Du nouveau roman qu'avait commencé à écrire Francesco Biamonti, il ne nous reste que vingt-neuf pages dactylographiées, de nombreuses variantes, un titre hypothétique, et deux brefs entretiens, qui exposent les grandes lignes de l'inspiration et la trame de l'œuvre. C'est avec une certaine perplexité philologique, qu'à présent nous pouvons lire cette ébauche de récit dans une petite édition posthume, chez Einaudi ${ }^{1}$. Le mérite de l'entreprise éditoriale, du moins pour ce qui est de la critique, n'est pas tellement de présenter au public une poignée de pages retrouvées, mais de pouvoir vérifier sur un objet largement provisoire une lecture plus générale de l'œuvre biamontienne. Car Il silenzio, qui a été conçu pendant les dernières années de la vie de l'auteur, présente une physionomie unique, et en même temps sans surprises.

Pour qui connaît L'Ange d'Avrigue, Vent largue, Attente sur la mer, Les Paroles la nuit ${ }^{2}$, hormis la déception d'une lecture trop tôt terminée, $I l$

1. Biamonti F., Il silenzio, Turin, Einaudi, 2003, Le Silence, traduit par Carole Walter, Lagrasse, Verdier "Terra d'altri», 2005. Il existe aussi une traduction partielle par Pozzoli M., La pensée de Midi, $\mathrm{n}^{\circ}$ 3, 2000.

2. Biamonti F., L'Ange d'Avrigue (L'angelo di Avrigue, 1983), traduit par Ph. Renard, Lagrasse, Verdier "Terra d'altri», 1990; Vent largue (Vento largo, 1991), traduit par B. Simeone, Lagrasse, 
silenzio n'apparaît pas comme une œuvre inachevée, pas plus que les autres romans publiés du vivant de l'auteur. Bien évidemment, s'il en avait eu la possibilité, Biamonti aurait continué à y travailler, et pourtant ce qui reste dans ces vingt-neuf pages résume l'ensemble de sa poétique, dans une sorte d'icône, ou de mini-roman. Il est difficile de savoir si la maladie a joué un rôle structurant, en poussant l'auteur à condenser en très peu d'espace (et de temps) un récit qui, dans d'autres circonstances, aurait pu se développer différemment. En revanche, il semble certain que Il silenzio se présente comme une sorte de métonymie exemplaire, un lieu privilégié où les exercices de lecture critique seront valables pour l'ensemble de l'œuvre de Biamonti.

On disait plus haut que le texte à analyser demeure également sans surprises. Un peu comme Cézanne a peint la montagne Sainte-Victoire avec une obsession cognitive qui fait pendant à l'indétermination phénoménale du paysage, à chaque saison et sous chaque perspective, Biamonti a, pour ainsi dire, écrit et publié quatre fois le même roman, et il était de nouveau en train de s'y essayer. À chaque roman terminé, c'est comme si l'auteur devait absolument recommencer, en déplaçant son angle d'observation de quelques degrés. En définitive, les personnages, thématiques, lieux et dynamiques narratives demeurent inchangés, mais l'écrivain, comme il le ferait s'il se trouvait en plein air en face d'un paysage, se remet à observer et à décrire, en vue de combattre le désagrément de l'ineffable, de l'inépuisable, avec une perspicacité phénoménologique héritée de Merleau-Ponty ${ }^{3}$. Italo Calvino à écrit ceci :

Ogni volta che ho provato a descrivere un paesaggio, il metodo da seguire nella descrizione diventa altrettanto importante che il paesaggio descritto: si comincia credendo che l'operazione sia semplice, delimitare un pezzo di spazio e dire tutto ciò che si vede; ma ecco che subito devo decidere se ciò che vedo lo vedo stando fermo, come di solito stanno i pittori, o almeno stavano, al tempo in cui i pittori dipingevano paesaggi dal vero - tempo che è durato tre secoli a dir tanto, cioè una fase molto breve della storia della pittura - oppure lo vedo spostandomi da un punto all'altro entro questo pezzo di spazio in modo da poter dire quello che vedo da punti diversi, cioè moltiplicando i punti di vista d'uno spazio tridimensionale. [...] Ma bisogna subito dire che mentre io scorro nel paesaggio per descriverlo come risulta dai diversi punti del suo spazio, naturalmente è anche nel tempo che scorro, cioè descrivo il paesaggio

Verdier "Terra d'altri», 1993; Attente sur la mer (Attesa sul mare, 1994), traduit par F. Maspero, Paris, Le Seuil, 1996; Les Paroles la nuit (Le parole la notte, 1998), traduit par F. Maspero, Paris, Le Seuil, 1999.

3. Pour connaitre Biamonti en tant que lecteur passionné de la phénoménologie de MerleauPonty, voir Biamonti F., «È morto Maurice Merleau-Ponty», Il Giornale dell'Unione Culturale Democratica di Bordighera, avril-mai 1961. 
come risulta nei diversi momenti del tempo che impiego spostandomi. Perciò una descrizione di paesaggio, essendo carica di temporalità, è sempre racconto : c'è un io in movimento che descrive un paesaggio in movimento, e ogni elemento del paesaggio è carico di una sua temporalità, cioè della possibilità d'essere descritto in un altro momento presente o futuro... ${ }^{4}$

La multiplication des points de vue, la temporalité suggérée par le mouvement physique, la précarité de la perception spatiale et les potentialités cachées constituent des coordonnées des plus efficaces dès lors qu'il s'agit de s'orienter dans l'écriture de Biamonti. Il convient d'ajouter à cela une angoisse subtile qui, d'après Calvino, ne fait qu'un avec les limites mêmes de l'écriture:

Descrivere vuol dire tentare delle approssimazioni, che ci portano sempre un po'più vicino a quello che vogliamo dire, e nello stesso tempo ci lasciano sempre un po' insoddisfatti, per cui dobbiamo continuamente rimetterci ad osservare e a cercare come esprimere meglio quel che abbiamo osservato ${ }^{5}$.

Dans son compte rendu de la première édition de L'Ange d'Avrigue, Calvino lui-même écrivit à propos du roman qu'il y a «des romans-paysage de la même façon qu'il y a des romans-portrait. Celui-ci vit page après page, heure après heure, de la lumière du paysage rude et escarpé de l'arrière-pays ligurien, dans son extrême pointe d'Occident, à la frontière avec la France ${ }^{6} »$.

Calvino parle de "romans-paysage», et non de "romans de paysage», car il ne pense pas à une présence répétitive de paysages enchâssés dans un tissu narratif: ce qui signifie que les romans de Biamonti sont bien édifiés comme des paysages, d'après une logique qui n'est pas celle de la narration linéaire, mais qui se développe avec une structure par taches et par mouvement de taches, dépourvue d'un centre fixe, sans début ni fin, de manière inachevée, changeante, et pourtant toujours égale à elle-même. C'est bien ce qui provoque la sensation que les romans prétendument "achevés» demeurent en quelque sorte ouverts, tandis que le roman réellement inachevé conserve une harmonie interne, renvoyant à un mouvement circulaire. De la première à la dernière page, Biamonti nous familiarise avec une sorte d'absence de marges, un continuum spatio-temporel où

4. Calvino I., «Ipotesi per una descrizione», Italo Calvino. Enciclopedia: arte, scienza e letteratura, M. Politi éd., Milan, Marcos y Marcos, 1995, p. 96-97.

5. Calvino I., "Osservare e descrivere», Italo Calvino, op. cit., p. 88.

6. In Biamonti F., L'angelo di Avrigue, Turin, Einaudi, 1983: : Ci sono romanzi-paesaggio così come ci sono romanzi-ritratto. Questo vive, pagina per pagina, ora per ora, della luce del paesaggio aspro e scosceso dell'entroterra ligure, nell'estremo suo lembo di Ponente, al confine con la Francia.» 
le début et la fin sont des opérations arbitraires, nous accoutument à un rythme syncopé qui fait un avec l'entrelacement homme-paysage ${ }^{7}$. Il est en effet impossible de saisir l'œuvre de Biamonti si l'on fait abstraction de sa conception de la nature. Au cours d'un entretien sur $I l$ silenzio, il faisait remarquer ceci :

Potenzierò la trama ma abbandonerò la natura come consolazione. Nei miei romanzi la natura va dalla vita alla morte, dalla morte alla vita, è completamente metamorfica, lo spazio è inficiato, il tempo è malato e il mondo è su un abisso. Però molti si consolavano con le mie descrizioni delle nuvole, del cielo, del mare. Ora non voglio più offrire questa consolazione, voglio che questo diventi un ulteriore pungolo all'angoscia che investe tutta la nostra coscienza ${ }^{8}$.

\section{Mais seulement quelques mois plus tard, il était revenu à son premier pro- pos:}

Ancora di più è sentito il rapporto con gli elementi naturali che vengono a sostituire quelli ideologici; il paesaggio diventa coscientemente consolatorio, ma in maniera indiretta: come riflessioni sul paesaggio, sulla funzione che il paesaggio ha avuto, da Cézanne ai giorni nostri, una riflessione sulla natura non come spettacolo diretto ma come meditazione sugli aspetti della vita, in senso leopardiano...?

La consolation constitue bien une thématique centrale, mais Biamonti développe une réflexion méta-littéraire portant sur le paysage, dans un article mal connu qu'il écrivit en français:

Tout change avec le XX⿳亠口冋 siècle: la nature devient indifférente, lointaine et plonge dans un désordre cosmique. [...] Toute la littérature existentialiste est traversée par cette conception d'une nature qui ne signifie plus rien, qui n'est plus que le «corrélatif objectif» de l'état d'âme de l'homme. Et avec elle, toute la lignée de la littérature ligurienne et de la côte française de la Méditerranée où la nature fournit des objets qui servent à établir l'état d'âme de celui qui regarde. Ce mélange d'émotivité humaine et de données naturelles commence avec Cézanne. Avec Cézanne, la nature devient non seulement le "corrélatif» de l'état d'âme du peintre mais aussi la fondation de l'émotion même. Il y a cette objectivité de l'émotion qui se transfere dans les choses tout en traçant une sorte d'autoportrait ${ }^{10}$.

7. Sur le paysage chez Biamonti, voir Bertone G., Letteratura e paesaggio. Liguri e no: Montale, Caproni, Calvino, Ortese, Biamonti, Primo Levi, Yehoshua, Lecce, Manni Editore, 2001; Mallone P., "Il paesaggio è una compensazione». Itinerario a Biamonti, Gênes, De Ferrari Editore, 2002.

8. Entretien d'A. Viale (21 septembre 1999), in Biamonti F., Il silenzio, op. cit., p. 38.

9. Entretien de M. Camponovo (8 juin 2000), in Biamonti F., Il silenzio, op. cit., p. 42.

10. Biamonti F., "Le percept de nature dans la poétique contemporaine à partir des "Correspondances» baudelairiennes", J-P. Manganaro, G. Passerone, C. Bobas, A. Marino, N. Gailius éd., Réalités et temps quotidien. Matériaux de la culture italienne contemporaine, Paris, L'Harmattan, 2001, p. 15-20, et particulièrement p. 16. 
La perception de ces rapports qu'entretiennent hommes et nature demeure cruciale quand il s'agit de remonter aux origines de l'écriturepaysage biamontienne. L'état d'âme de l'écrivain, ou du personnage, se cristallise sur un fragment de paysage, sur une réalité phénoménale qui absorbe dans sa traduction verbale le regard plein d'émotion porté sur cette même réalité. Il s'agirait donc d'un autoportrait psychologique objectivé. Mais cette réalité, d'une manière circulaire, devient à son tour fondement de l'émotion. Autrement dit, le paysage n'est pas le simple reflet d'un état d'âme, ou un réactif qui aide l'écrivain à colorer l'émotion, mais il coexiste en harmonie avec elle, comme si hommes et paysages agissaient selon des dynamiques similaires et inéluctables ${ }^{11}$.

La palette biamontienne reste toujours la même: "Le fondamental, pour nous qui habitons sur les rives de la Méditerranée, c'est le rocher, les oliviers, le ciel étoilé, la nuit, l'amour, la mort, l'amitié ${ }^{12}{ }^{2}$. Plus qu'un paysage compénétré d'émotions, Biamonti préfere mettre en scène un monde d'émotions primaires s'organisant en paysage, et notamment sur le paysage: la structure narrative, les aspirations des personnages, les sentiments se distribuent d'abord dans l'espace, et seulement ensuite dans le temps. Et c'est bien l'imitation dans l'écriture des caractéristiques morphologiques des lieux qui aboutit à cette spatialisation du récit: des taches et des dynamiques de taches à partir desquelles homme et nature s'organisent dans une sorte d'écosystème, une logique naturelle et verbale qu'on reconnaît quand on survole le texte ${ }^{13}$.

Il s'agit ici d'une écologie textuelle et mentale, où l'opposition merhomme-voyage d'un côté, et terre-femme-maison de l'autre, devient l'axe sur lequel se structure l'intégralité du récit, selon une antinomie omniprésente chez Biamonti ${ }^{14}$. L'interpénétration de l'esprit et du paysage est si forte que la psychologie humaine est à la fois conséquence directe et inter-

11. «Paysage et âmes, non pas un simple paysage de l'âme» ("Paesaggio e anime e non un semplice paesaggio dell'anima»), comme l'a dit Conte G., "Donne provate dalla violenza e ragazzi sulla via della droga», Avanti!, 18 février 1983.

12. Biamonti F., «Le percept de nature», art. cit., p. 17.

13. Sur le paysage comme fonction narrative, voir nos articles «Il paesaggio arcipelagico della «Navigatio Sancti Brendani»», Studi celtici, n 1, 2003, p. 159-173; "Forme dello spazio nelle chansons de geste. Il caso della lassa similare», Quaderni di filologia romanza, Bologna, ${ }^{\circ} 16$, 2003, p. 217-229.

14. Sur cette opposition chez Biamonti, étudiée d'après les catégories du lisse et du strié de Deleuze et Guattari, voir Lanslots I., "Ascoltare il mare come una liturgia o prendere il volo", B. Van den Bossche, M. Bastiaensen, C. Salvatori Lonergan éd., "... E c’è di mezzo il mare» : lingua, letteratura e civiltà marina, Actes du XIV Congrès AIPI, Spalato, 23-27 août 2000, 2 vol., Franco Cesati Editore, 2002, vol. 2, p.313-325. 
prétation authentique des «lois» du lieu. C'est le cas de l'ambivalence des protagonistes, qui basculent constamment, ne savent s'il doivent partir ou rester, s'égarer dans le présent ou se retrouver dans le passé des ancêtres, hésitent entre une mer à traverser et une montagne à travailler. C'est le reflet logique du paysage ligurien, sa dialectique et son équilibre instable, un paysage qui produit invariablement des personnages de marins rongés par une nostalgie de la terre ferme, et de femmes du continent qui songent à la mer. Le tout est animé par un désir ardent de calme et d'unité, mais au bout du compte cette mécanique existentielle nie la rencontre, l'échange, et engendre l'inéluctable silence entre les sexes. Calvino, dans le compte rendu cité plus haut, disait du personnage masculin: "Quatre personnages féminins, chacun avec son obsession, croisent ses pas; mais les solitudes, dans la somme, ne s'annulent pas ${ }^{15}{ }^{\prime}$. Car les opposés ne s'annulent pas, ils se nuancent seulement sur les marges, tout en conservant des éléments ambigus qui compliquent leur profil, mais concèdent à l'esprit un équilibre précaire entre séduction et refus.

Chez Biamonti, le thème de la séduction est central, mais dans Il silenzio il est traité avec une auto-conscience et une dureté sans appel. À l'appui de notre propos sur la fonction structurante du paysage, on observera que la séduction trouve son espace d'expression en se rivant sur une double géographie, à la fois concrète et intime. Dans un décor naturel polarisé entre mer et terre ferme, dans un cosmos préalablement établi, à l'exception des modalités de sa fin annoncée, tout porte à croire qu'il n'y a plus de place pour le libre arbitre, que la séduction n'est plus un art raffiné, mais une force d'attraction primaire, déterministe, décharnée, réduite a sa plus simple expression, comme les terres avares qui semblent engendrer l'action.

L'incipit de Il silenzio est très simple: la rencontre d'un homme et d'une femme, quasiment inconnus l'un à l'autre dans un lieu naturel. Sur leur compte on ne sait rien. La femme cherche des fleurs, elle parle à l'homme, l'homme à son tour parle de l'endroit où ils se trouvent. C'est le strict nécessaire pour entamer un dialogue, et pour montrer les premiers moments d'une sympathie qui amènera à autre chose: "Se non ci fosse quest'amica che mi aspetta mi fermerei volentieri", dit la femme, et encore: "Un giorno, torno. Se capita in paese mi venga a trovare» (p. 5-6). L'homme descend au village mais la femme n'est pas chez elle. Plus tard, il la rencontre dans la rue, et pour la première fois ils se présen-

15. In Biamonti F., L'angelo, op.cit., "Quattro personaggi di donne, ognuna con una sua ossessione, incrociano i suoi passi; ma le solitudini sommandosi non si annullano». 
tent. La scène se déroule d'une façon linéaire, jusqu'au moment où les intentions de la femme deviennent explicites. Le chapitre commence avec une ouverture sur le paysage, qui prépare l'action tout en composant le substrat logique de la narration: "Lei era tornata a cercare qualche fiore, ma in quel periodo di riposo della terra, la linfa, rintanata nei vecchi alberi, non aveva ancora preso a sbocciare nell'oro delle mimose. Nei giorni brevi, di luce necessaria per vivere, venature rosa si allungavano nei rami dei lentischi» (p. 10).

D'autre scènes de séduction chez Biamonti commencent de la même manière: une femme enveloppée de végétaux et affectée par leur toucher, et un homme qui parle des lieux, comme dans Vent largue:

Sabèl toccò le foglie di una waldorf; le restò sulle dita una sorta di polvere.

- Si coprono di polvere per non traspirare, - egli disse.

- Se continua questo secco non riusciranno a portare il fiore ${ }^{16}$.

On reconnaît ici une fonction narrative essentielle: la rencontre homme-femme se déroule dans un jardin qui mélange beauté et danger, fécondité et aridité. La scène semble construite sur un socle archétypique, adamique presque, et la séduction semble répondre à un schéma fixe qui transcende la volonté des individus car, au bout du compte, c'est la même vieille histoire qui se répète depuis toujours ${ }^{17}$. Dans Vent largue, Sabèl est tachée et presque souillée par le végétal, dans Il silenzio Lisa est blessée par une plante:

Si mosse tra i lentischi; uno spuntone le escoriò una gamba, sopra il ginocchio. Vi batté la luce di una nuvola bianca. Passavano delle nuvole, il cielo sembrava scivolare. Solo verso la montagna era di un azzurro rugoso. [...] Lei gli chiese dell'acqua ossigenata, Edoardo andò a prenderla, stappò la bottiglia e gliela porse. Lei sedé e si alzò la gonna. - Non si volti, può guardare, - disse. Versò l'acqua sulla graffiatura; si chinò a guardarla. Le biancheggiò sopra la fronte una ciocca di capelli. Tinta ad arte? Le altre erano di un castano lieve. Si alzò. - Ecco, sono pronta.

- Perché tanta premura?

- Tanto lei non mi guarda. (Il silenzio, p. 10-11)

Comme dans Perceval de Chrétien de Troyes, la plaie et la contemplation de la plaie sont la mise en images d'une pulsion sexuelle sans équivoques. La séduction est ici une séduction primaire, presque brutale de par sa

16. Biamonti F., Vento largo, Turin, Einaudi, 1991, p. 4.

17. Sur le jardin comme lieu archétypique de séduction, la référence va bien évidemment au poème de Jaufré Rudel Quan lo rius de la fontana, que Biamonti connaît très bien (voir l'entretien à Biamonti par Improta F., "Grandi, sconcertanti silenzi", La Gazzetta di San Biagio, n³3, octobre 2002), et à la tradition romane de la pastourelle. 
nature explicite, mais ce qui compte surtout est le parallèle entre la lumière blanche qui effleure le genou de la femme et la mèche teinte qui blanchit sur son front. L'élément chromatique et lumineux crée un lien subtil entre le corps et le paysage. La clarification est réciproque: la beauté de la lumière est peut-être fallacieuse, tout comme la mèche peut être trompeuse, car colorée à dessein. Dans le Jardin, Adam est donc mis en garde par de subtiles allusions: le ciel s'écoule, les nuages passent comme les signes d'une histoire éphémère, et la femme, fugace elle aussi, se résout à partir en toute hâte. Cette double "premura", au goût des choses qui finissent, met à nu le point faible d'Edoardo qui, comme tous les personnages masculins de Biamonti, résiste, déjà vaincu, à l'automne du monde.

Et pourtant la séduction est efficace, car dans une terre où tout est nu, dépouillé, c'est justement la nudité de la peau qui guide l'acte de séduction, suggère sans médiation l'union des corps. Dans un univers où les solitudes ne s'annulent pas, où la terre touche la mer sans vraiment la rencontrer, où hommes et femmes incarnent une vérité manichéenne, l'art de la séduction est superflu. Il suffit d'une autre rencontre pour passer à l'action, et amorcer la fin:

Lisa tornò tre giorni dopo, di sera. Il viola ardeva sui dirupi, li univa al mare, avviluppava l'uliveto. L'aria scuoteva i rami, ma lei non sembrava avere freddo; solo più fondo il blu degli occhi, più nuda la pelle nuda. [...] Di quella sera rimase stagliata solo lei che si stendeva nella penombra, che dava, che esigeva. Ma non erano completamente soli nella sera. Si era impossessato di lei un essere strano e ne diceva i desideri con frasi secche e ondosi gemiti. E da che avevano riacceso si era dissolto. (Il silenzio, p. 13-14).

Il n'y a plus rien de l'innocence primitive du jardin. Seul subsiste, cru, froid, le paysage: en dépouillant d'un seul mouvement les branches et la peau, il transmet à Lisa la sauvagerie du vent. Les «frasi secche e ondosi gemiti» sont un paysage ligurien dans la pénombre, un paysage du corps qui reflète les terres arides et la mer agitée, un chiasme verbal et spatial qui résume, dans la figure rhétorique de la rencontre-séparation, toute la poétique biamontienne. Mais si le vent emprunte sa voix au délire nocturne de Lisa, c'est encore une fois la lumière qui soutient le discours amoureux, et le violet du crépuscule qui préfigure l'union des corps: non seulement la couleur enlace la mer et les escarpements, autrement dit le féminin et le masculin, mais elle brûle sans chaleur, comme la passion froide d'une Lisa qui brûle ardemment, mais qui se brûle dans l'espace d'une nuit. La lumière et ses variations forment un signal précieux pour le critique, car c'est à la lumière que Biamonti attribue une fonction allusive et intra-textuelle. C'est le cas de ce passage révélateur du roman, où l'auteur réitère ce 
modèle de connexion entre le corps de la femme et le paysage:

Il mare di là dai dirupi tentava il fiabesco: si squamava d'oro e incorniciava un paese aggrappato a picco. «Ma non mi prendi, - egli pensava, - la tua seduzione la conosco troppo; ci sovrasta». Cominciava a ricordare certe sere e albe e giorni, sconfinata memoria, da cui lei lo scosse.

- Sembra un braciere.

- Ancora mezz'ora e si spegne, - lui disse.

[...] Il dorato del mare, che prima si rifletteva sull'ampia scollatura della donna, sulle stille di perla che scendevano fino al seno, crollava senza alcun rumore nel blu cupo e nella cenere. La sera si avviava verso sentieri impervi. Ma nel ritorno, a notte fonda, cambiò di colpo. Lei denudava le gambe e le apriva, rovesciava la testa come presa da un grande sonno. (Il silenzio, p. 18-19)

La mer et la femme sont liées (non pas assimilées) par l'intermédiaire de la lumière au coucher du soleil, et toutes deux, en vrai palimpseste, deviennent des points d'attraction pour un ancien marin qui se méfie. L'or qui coule sur la poitrine de Lisa confere au passage un caractère féerique et ensorcelant, mais en fin de compte il est seulement une «fable». Pareillement, Lisa est un objet de désir fabuleux et fugace, une belle chose qui «in mezz'ora [...] si spegne». Ne subsiste que la cendre, et encore: la femme mène Edoardo vers une expérience quasi dantesque, qui gâche à jamais leur lien:

Entrarono in un piccolo atrio: aveva due scale, una saliva e l'altra scendeva nei sotterranei. C'era un tavolino con un registro e un guardaroba.

- Dovete darmi il nome e uno pseudonimo, - la donna disse.

Li accompagnò di sopra, in un gorgo di musica, in una sala orlata di divani, con luce a lampi che feriva gli occhi e svelava gente che si accoppiava. In mezzo alla sala qualcuno si ostinava a danzare.

Sedettero accanto a una coppia che era ancora alle carezze. Lei aveva un volto chiaro e ossuto.

- Che cercate?

- Emozioni, - Lisa disse.

- Siete arrivati un po'tardi.

- Qualcosa faremo.

Si sentivano lamenti simulati, qualche grido. «È un fuoco talmente finto, - lui pensava, - che non produce cenere. Un piccolo inferno addomesticato». (Il silenzio, p. 30-31)

Cet enfer domestiqué représente le dernier acte d'une histoire dont le corrélatif objectif est la mer qui «tenta il fiabesco». Sa séduction n'est qu'une lumière de surface, un feu feint qui ne laisse pas de cendre. Lisa dit à Edoardo : "Forse sono stata un libro troppo aperto " (p. 31), et elle lui pose ensuite une question qui fait office de conclusion au roman inachevé: «Perché non mi hai fermata?» (p. 32). Mais il n’y a pas de réponse, il y a le 


\section{Matteo Meschiari}

silence du titre, un silence que Lisa avait prévu:

- Non c'è più bisogno di parole, - diceva.

- Di che cosa c'è bisogno?

- Di mettere qualcosa di seducente, di forte sopra le ferite, in modo da renderle indolori e invisibili.

- Ci possiamo provare.

- Torno ancora per l'ultima volta sullo stesso argomento. Me ne vorrai scusare. Ti pare giusto morire per un'ideologia al tempo della sua morte?

- No, non mi pare giusto. Ma non c'è più tempo per nessuna fede.

(Il silenzio, p. 19)

Recouvrir les blessures par quelque chose de séduisant, ou, comme elle le dira plus tard, poser des images "gioiose [...] contro l'angoscia che avanza» (p.31), c'est une idée commune à Biamonti et à Lisa, tous deux néanmoins conscients de la séparation. C'est pour cette raison qu'Edoardo se trouve dans l'impossibilité de répondre à la question "perché non mi hai fermata?", pour cette raison encore que malgré ses précédents propos, Biamonti n'a pas été en mesure d'effacer la composante naturelle du roman qu'il venait de commencer:

Alors, le paysage, à quoi ça sert? Ça sert de consolation, comme disait Camus, comme les tablettes que les frères du Moyen Age mettaient devant les condamnés à mort sur le chemin qui les menait à l'échafaud. Peut-être c'est ça: il y a un silence du monde, mais la tâche de l'écrivain, c'est de rendre ce silence, faire en sorte que le monde parle. Faire parler le monde dans un monde de la chute des idéologies et de l'éloignement du divin, c'est difficile : il faut apprendre à écrire en regardant le rocher vers le ciel étoilé. ("Le percept de nature», p. 17)

Lisa invoque ainsi le silence, et demande de jeter un voile séduisant, fort et gai sur les blessures, sur l'angoisse qui monte, sur la chute des idéologies. Le voile c'est la chair, la chair de la femme et la chair du paysage. Et Biamonti, par le biais de son personnage Edoardo, écoute la femme et la satisfait, en récréant une confusion parfaite entre le corps de Lisa et le «corps» de la Ligurie:

Lasciarono il mare, che tanto non si vedeva; entrarono in vallata; $s$ 'inerpicarono, passarono accanto al paese addormentato, andarono a casa di Edoardo. Contraddicendosi, facendo il contrario di ciò che si erano proposti, parlarono sino a giorno fatto. Lei si spogliò che il cielo era di cobalto, di un azzurro che non riusciva a fondersi né con le rocce né con le montagne; gli ulivi vi si specchiavano. La campagna in quel momento non era fatica e pena, ma qualcosa d'altro. «Il suo seno è splendido ", egli pensava. Si proiettava sulla parete come gli ulivi sul cielo. La luce entrava senza fiatare, tratteneva il respiro. Era una giornata ferma, di rami immobili. Lo zirlo dei tordi nell'uliveto e il fruscio degli abiti, che cadevano, suonavano come cristallo.

(Il silenzio, p. 19-20)

À l'instar d'Edoardo et Lisa, Biamonti se contredit, et renonce au silence 
pour parler du paysage. Car le paysage, dont la destruction nous est pourtant annoncée, est bien la vraie, la dernière séduction. Exclure la terre, nier le "caractère primordial de la terre dans l'aventure humaine» ("Le percept de nature», p. 18), cela reviendrait à perdre un chemin d'accès au réel. C'est ce qui arrive, d'une manière fort symbolique, à la mèche blanche de Lisa: vient le jour où elle décide de se teindre les cheveux, et Edoardo remarque alors que désormais elle a effacé « una strada, un invito all'intimità del suo corpo" (Il silenzio, p. 22).

Chez Biamonti, l'amour arrive toujours en retard, dans le désenchantement, mais il arrive aussi comme un paysage, pour soulager la souffrance d'une existence vide et inutile. L'amour est cette lumière blanche qui glisse sur le genou de Lisa, l'or qui refroidit sur sa poitrine, la nature qui touche à sa fin. Biamonti dit: "Il faut se river à cette terre comme à une proie, comme à la seule consolation possible, avec sa dureté et malgré la fatale séparation» («Le percept de nature», p. 19). Ce qui revient à vivre le présent avec fatalité, s'agripper à l'amour pour les choses, sans les nier, et garder seulement de la séduction son essence ultime: le besoin d'une proie. 